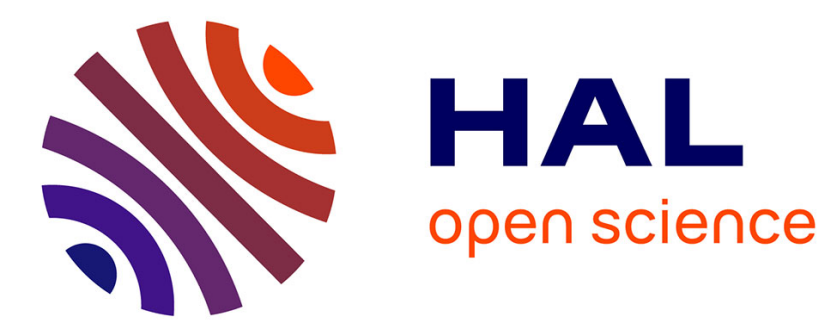

\title{
Un exemple fondateur de collaboration interdisciplinaire : 9 evenings : theatre and engineering \\ Clarisse Bardiot
}

\section{To cite this version:}

Clarisse Bardiot. Un exemple fondateur de collaboration interdisciplinaire : 9 evenings : theatre and engineering. Ligeia, dossiers sur l'art, 2015, 137-140, pp.79-87. 10.3917/lige.137.0079 . hal-02339182

\section{HAL Id: hal-02339182 \\ https://hal.science/hal-02339182}

Submitted on 30 Oct 2019

HAL is a multi-disciplinary open access archive for the deposit and dissemination of scientific research documents, whether they are published or not. The documents may come from teaching and research institutions in France or abroad, or from public or private research centers.
L'archive ouverte pluridisciplinaire HAL, est destinée au dépôt et à la diffusion de documents scientifiques de niveau recherche, publiés ou non, émanant des établissements d'enseignement et de recherche français ou étrangers, des laboratoires publics ou privés. 


\title{
UN EXEMPLE FONDATEUR DE COLLABORATION INTERDISCIPLINAIRE : 9 EVENINGS : THEATRE AND ENGINEERING
}

\author{
Clarisse Bardiot
}

De nombreux spectacles impliquant des technologies numériques nécessitent la contribution d'ingénieurs, voire de chercheurs de haut niveau, spécialisés dans l'intelligence artificielle, la robotique, les interfaces humain-machine, la programmation informatique, etc. C'est ainsi que de nouveaux collaborateurs partagent le plateau avec les régisseurs et les différents interlocuteurs techniques traditionnels. Les métiers de ces derniers en sont parfois bouleversés, car leurs savoir-faire et leurs outils peuvent être radicalement mis en cause. Souvent, cette collaboration dépasse le cadre de la technique et engage l'équipe artistique dans son ensemble. Des ponts doivent alors être créés, des modes de dialogue se constituer. Rien de simple, un pari nullement gagné d'avance, remis sur le métier à chaque nouveau projet. Avec un risque : que la technologie ne prenne le pas sur le projet artistique, que le spectacle ne devienne une «démonstration» des potentialités de telle ou telle solution technologique au détriment du propos artistique et dramaturgique.

Un exemple précurseur de collaboration artistes/ingénieurs dans le domaine des arts de la scène est la manifestation 9 Evenings, Theatre \& Engineering ${ }^{l}$. Presque cinquante ans plus tard, les problématiques alors rencontrées demeurent d'actualité.

À New York, en 1966, dix artistes (John Cage, Lucinda Childs, Öyvind Fahlström, Alex Hay, Deborah Hay, Steve Paxton, Yvonne Rainer, Robert Rauschenberg, David Tudor et Robert Whitman) oeuvrent avec une trentaine d'ingénieurs de Bell Labs, dont Max Mathews, Bela Julesz, Peter Hirsch, Fred Waldhauer et Per Biorn. Ensemble, ils créent dix performances présentées pendant neuf soirées. Si les critiques sont négatives - et c'est un euphémisme - le succès public est au rendez-vous : 10000 personnes assistent aux représentations, parmi eux de nombreux artistes de la scène new-yorkaise, dont Andy Warhol. Intitulée 9 Evenings : Theatre and Engineering cette manifestation est aujourd'hui considérée comme un exemple de collaboration interdisciplinaire.

Billy Klüver est à l'origine de ce projet. Né en 1927 et titulaire d'un Ph.D. en génie électrique de l'Université de Californie à Berkley, il est alors ingénieur au département des

1. Cette recherche a été effectuée dans le cadre du programme de chercheur en résidence de la Fondation Daniel Langlois (Montréal) en 2005 et publiée sur internet en 2006. Cet article est une version revue et augmentée des textes mis en ligne en 2006. Une analyse plus précise de chaque performance est disponible sur le site Internet de la Fondation Daniel Langlois. http://www.fondation-langlois.org/flash/f/index.php? NumPage=571 Consulté le 8 septembre 2014 . 
communications dans les laboratoires de recherche de la société Bell Telephone. Pendant la première moitié des années 1960, Billy Klüver est conseiller technique auprès de différents artistes (Andy Warhol, Jean Tinguely, Robert Rauschenberg...).

Fort de ces expériences et de ces multiples rencontres, Billy Klüver décide fin 1965 de préparer un événement d'envergure intitulé Stockholm Festival for Art and Technology. Visions of The Present, qui aurait dû se dérouler au Musée des techniques de Stockholm. Il souhaite trouver « de nouveaux moyens d'expression pour les artistes [...] et connaître leur position par rapport à une société qui envoie des hommes sur la Lune $»^{2}$. Le programme initial comprend des conférences (sont notamment prévues des interventions de Marshall McLuhan, de John Pierce et de Buckminster Fuller), des expositions et des performances.

La manifestation n'aura finalement pas lieu à Stockholm, pour des raisons de financement de la part des sponsors américains. Au dernier moment, la décision est prise de présenter les performances à l'Armory à New York, lieu où avait eu (pure coïncidence) la célèbre exposition, The Armory Show, en 1913. Les conférences et les expositions sont abandonnées ; l'événement change de titre. Il devient 9 Evenings : Theatre and Engineering et se déroule du 13 au 24 octobre 1966. Chaque soirée présente deux à trois performances, ce qui permet de jouer chacune d'elles deux fois.

Les artistes de 9 Evenings sont liés par des collaborations antérieures au festival. Yvonne Rainer, Deborah Hay, Alex Hay, Lucinda Childs et Steve Paxton sont issus du Judson Dance Theater, un collectif créé fin 1962 à New York qui pose les bases de la postmodern dance. Robert Whitman et Öyvind Fahlström fréquentent ce groupe, composé de chorégraphes, de plasticiens et de musiciens. Les membres du Judson Dance Theater sont fortement influencés par deux figures importantes : John Cage et Merce Cunningham. Le studio de Cunningham a formé presque tous les chorégraphes du collectif, et certains d'entre eux, dont Deborah Hay et Steve Paxton, ont été danseurs dans sa compagnie. Robert Rauschenberg, assisté d'Alex Hay, en est le scénographe. Il rejoint par ailleurs le Judson Dance Theater en 1963. David Tudor est l'interprète principal des œuvres de Cage, dont plusieurs sont composées pour les chorégraphies de Cunningham. Le plus souvent, notamment pour les artistes issus du Judson Dance Theater, l'expérience en matière de technologie est très réduite. Elle consiste en

2. «9 Evenings : Theatre and Engineering 》 (manuscrit), in Harriet DeLong, Experiments in Art and Technology, 1966-1967 (1972-1973), Box 2, Experiments in Art and Technology, Records, 1966-1993, Research Library, The Getty Research Institute, Los Angeles, California (940003). 
l'insertion de films, l'utilisation de micros et la réalisation de montages sonores pour des performances.

Les ingénieurs impliqués dans 9 Evenings sont tous issus, à quelques exceptions près, des laboratoires Bell (Murray Hill, N.J., États-Unis), une entreprise américaine dotée d'un département de recherche parmi les plus importants au monde à cette époque. On lui doit entre autres la mise au point du laser, des télécommunications par satellite ou encore du système informatique Unix. Plusieurs spécialistes de l'informatique sont sollicités dans le cadre de 9 Evenings : Bela Julesz, directeur du Sensory and Perceptual Processes Department développe le Computer Pictorial Data Processing; Cecil Coker, membre de l'équipe qui élabore le Synthetic Speech Computer; Max Mathews, alors directeur du Behavioral Research Laboratory, auteur des tous premiers programmes informatiques de numérisation et de traitement du son, ainsi que de composition musicale assistée par ordinateur. Les autres domaines de recherche scientifique représentés sont le traitement du signal sonore et l'acoustique (Pete Cumminski, Ken Harsell, Peter Hirsh, Manfred Schroeder), les lasers (Larry Heilos, Harold Hodges), la téléphonie mobile (Robby Robinson), la radio (Herb Schneider, Bill Kaminski), les systèmes de communications (Fred Waldauher), la chimie (Stretch Winslow, Tony Trozzolo), l'électronique (Witt Wittnebert, Per Biorn, Dick Wolff) et les hologrammes (Jim McGee).

Avec Billy Klüver, certains de ces ingénieurs ont déjà contribué à des projets artistiques : Max Mathews et Cecil Coker ont participé à Variations $V$ de Cage, sur une chorégraphie de Cunningham (1965), Harold Hodges à Oracle de Rauschenberg (1962-1965). Pourtant, la plupart des ingénieurs impliqués dans 9 Evenings ne sont pas ou peu au fait de l'art contemporain, et leur collaboration avec des artistes lors de ce festival est bien souvent leur première expérience en la matière. L'inverse vaut aussi pour les artistes, à l'exception de Rauschenberg, Cage et Tudor, qui développent un travail et une réflexion sur l'impact des technologies dans leur pratique artistique depuis plusieurs années. Ainsi, le 21 mai 1966, quelques mois avant 9 Evenings, à l'occasion d'une table ronde intitulée « The changing audience for the changing arts », John Cage commence ainsi son intervention : «Sommesnous un public pour l'art par ordinateur? La réponse n'est pas non ; c'est oui ${ }^{3}$. » Lecteur de McLuhan et de Buckminster Fuller, Cage est à cette époque très attentif aux développements des réseaux de télécommunication, à leur impact sur la société et sur l'art.

3. Cage John, « Diary: audience $1966 »$, in A year from monday, new lectures \& writings by John Cage, Londres, Marion Boyars, 1975 , p. 50. Édition originale : Calder and Boyars Ltd, 1968. 
Lors des premières réunions pour 9 Evenings — la première a lieu le 14 janvier 1966 — les artistes font part de leurs idées et imaginent une multitude de situations et d'objets : « un sonar Doppler pour capter les mouvements ordinaires du corps », « De nombreux (50? 100?) hautparleurs de faible puissance placés sur les murs ou autour de la salle, voire dehors. Chaque haut-parleur diffuserait un son différent», «une cloison d'air $»^{4}$, « des couleurs changeantes », « une chute de neige qui ne tombe pas ${ }^{5}$, « la possibilité d'avoir recours à Telstar, Early Bird ou une quelconque liaison transatlantique de communication par télévision ${ }^{6} »$, etc. Les ingénieurs étudient la faisabilité de ces demandes, font des contrepropositions et présentent des produits existants ou des axes de recherche possibles : Jim McGee suggère que «l'impulsion de muscles en flexion peut servir à déclencher des commutateurs (en lien avec le système sans fil proposé, par exemple) $»^{7}$. Lorsque les projets se précisent, certains ingénieurs travaillent plus spécifiquement avec un artiste (les binômes Tudor/Waldhauer, Rainer/Biorn, Cage/Coker...), tandis que d'autres ingénieurs se consacrent à la mise au point du dispositif général (le «TEEM»), ou font office de consultants. Ainsi, Lucinda Childs souhaitait poursuivre sa recherche sur le lien entre mouvement et objets (commencée lors de ses premières chorégraphies au Judson Dance Theater), en faisant en sorte que le mouvement des objets soit transformé en son, afin que le mouvement lui-même génère son propre accompagnement musical. Lors d'une rencontre avec Manfred Schroeder, alors directeur du laboratoire Acoustics, Speech and Mechanics chez Bell Labs, celui-ci lui propose d'utiliser un sonar: «On lui a proposé un dispositif qui reflétait les ondes ultrasoniques de son corps avant de les convertir en son audible. Je me suis inspiré de travaux que j'avais faits des années auparavant sur la stabilisation de systèmes de sonorisation et la rétroaction acoustique du bloc micro/haut-parleur, produisant dans certaines conditions des bruits de sifflement ou de chantonnement. Ces sons n'étaient pas très intéressants. Mais nous avions découvert que cela produisait toutes sortes de sons singuliers si on faisait ce qu'il fallait et s'il entrait des gens dans la pièce. Lorsque $\mathrm{M}^{\text {lle }}$ Childs a fait sa demande, je me suis souvenu de ces travaux. Ils nécessitaient un concept artistique pour être satisfaisants et agréables ${ }^{8}$. $\gg$ Le sonar Doppler, rebaptisé « Motion Music Machine », est par la suite mis en œuvre par Peter Hirsch, spécialiste du son en milieu marin. Ce n'est pas tant le mouvement du corps lui-même

\footnotetext{
4. Ce compte-rendu de la réunion du 13 février 1966 a été publié dans « Projects for Stockholm Festival ». In E.A.T. News 1 , n ${ }^{\circ} 2$, le $1^{\text {er }}$ juin 1967, New York, Experiments in Art and Technology, 1967, pp. 12-14.

5. Compte-rendu de la réunion du $1^{\mathrm{er}}$ mars 1966, in « Projects for Stockholm Festival », op. cit., pp. 15-16.

6. Billy Klüver, [Letter to J.R. Pierce]. 8 April, 1966. La fondation Daniel Langlois pour l'art, la science et la technologie, Collection de documents publiés par Experiments in Art and Technology, EAT C1-15, 15.

7. Compte-rendu de la réunion du $1^{\mathrm{er}}$ mars 1966, op. cit., p. 15.

8. «Art and science: two worlds merge », in Bell Telephone Magazine, vol. 46, n 6, nov./déc. 1967, pp. 15-16.
} 
qui génère le son que celui de trois seaux suspendus à un portique, auxquels Lucinda Childs donne différentes impulsions.

Des collaborations extérieures sont également nécessaires. Le projet d'Alex Hay - utiliser des capteurs pour amplifier et rendre audible des phénomènes physiologiques, comme le mouvement des muscles et les ondes cérébrales - demande la contribution d'un hôpital. Le titre de la performance, Grass Field, n'est pas une allusion à la nature mais un jeu de mot sur le matériel utilisé : les électrodes placées sur le corps d'Alex Hay sont fabriquées par la Grass Instrument Company, société ayant commercialisé l'électroencéphalogramme aux États-Unis.

Les ingénieurs qui contribuent à 9 Evenings mettent à profit leurs connaissances pour élaborer les différents instruments utilisés dans les performances. S'ils font appel aux dernières avancées de l'époque en électronique, ils ne font pas de découverte scientifique majeure, excepté les propriétés d'un phosphore lors de tests pour la performance de Robert Whitman. Aucun brevet n'est déposé à la suite du festival. L'innovation est ailleurs : il ne s'agit pas de partir de zéro, mais d'adapter des technologies existantes au contexte spécifique du spectacle vivant et de les mettre pour la première fois à la disposition d'artistes qui en proposent un autre usage, un détournement de leurs fonctions initiales. Ces derniers n'avaient (à quelques exceptions près) jamais pu avoir accès à de tels équipements, peu répandus, chers, et bien souvent non commercialisés auprès du grand public. C'est le cas des instruments utilisés dans les studios de télévision (micros sans fil, caméras et projecteurs vidéo), dans le secteur médical (électrodes), ou encore dans l'armée (caméras infrarouges). Ce changement de contexte permet de révéler le potentiel esthétique de ces instruments qui n'ont pas été développés dans une visée artistique. Ce détournement est très rarement à visée politique : malgré le contexte de la guerre du Vietnam, et le déroulement des performances dans un lieu dont la première destination est militaire, seul Öyvind Falsthröm opère dans son spectacle une mise en perspective critique des technologies.

Le changement de contexte suscite des adaptations dont Klüver est le premier à penser qu'elles auront des répercussions dans le monde industriel: miniaturisation de certains composants (par exemple l'insertion d'émetteurs FM dans les manches des raquettes de Rauschenberg), conception de batteries permettant l'autonomie de nombreux éléments du TEEM, etc. 
Malgré une apparence de collaboration idyllique entre artistes et ingénieurs ${ }^{9}$, les différents textes et témoignages recueillis montrent combien le dialogue ne va pas de soi. Comme le note Billy Klüver dans le programme de 9 Evenings, «cela n'a pas été aussi facile qu'il y paraît. Les artistes ont dû faire preuve d'énormément de patience face au rythme lent des ingénieurs. Et les ingénieurs ont dû se débrouiller avec le flou des artistes, ces derniers n'ayant rien à tenir dans leurs mains ou à travailler ${ }^{10}$. " Les artistes ont souvent l'impression d'être entraînés sur la pente des problèmes techniques au détriment du propos artistique, de ne pas maîtriser leur spectacle. De l'autre côté, les ingénieurs considèrent que les artistes ne prennent pas toujours en compte leurs contraintes spécifiques. L'éclatement géographique des uns et des autres, ainsi que le manque de temps, sont pour beaucoup dans ce constat, et les trois week-ends de travail commun dans un gymnase à Berkeley, en septembre, vont contribuer au rapprochement et à une meilleure compréhension des enjeux des uns et des autres.

C'est lors de ces rencontres qu'un ingénieur, Herb Schneider, prend conscience du problème suivant : «Ce qui m'a vraiment consterné, c'est que le 15 septembre personne ne savait ce qu'on ferait le 13 octobre, sauf de manière très générale. On a parlé pendant six heures avec chacun des artistes et on a ébauché ensuite les dessins et diagrammes des différentes combinaisons d'équipement dont chaque artiste aurait besoin. David Tudor demandait des fonctions que je n'arrivais pas à visualiser. Puis j'ai esquissé le dessin. Nous avons continué de préciser le tout et d'y apporter des corrections jusqu'à ce que je lui donne une forme finale. Je ne saisissais pas ce qu'il désirait avant de pouvoir le visualiser et il n'arrivait pas à me le communiquer, car il n'est pas habitué à visualiser des fonctions ${ }^{11}$. » Réalisés de fin septembre à début octobre 1966, les diagrammes permettent non seulement de préciser les projets de chacun des artistes, mais aussi de communiquer avec les ingénieurs afin de mieux coordonner les différents aspects techniques. Cette méthode permet (sauf dans le cas de la performance d'Yvonne Rainer) de s'abstraire du déroulement dramaturgique, de la succession des événements, pour élaborer un système. Les diagrammes sont l'outil qui permet d'articuler la pensée des uns et des autres, le pont à mi-chemin entre deux univers. Ils établissent un langage commun, une passerelle entre l'artiste et l'ingénieur, concrétisant, à peine un mois avant la date de la première représentation, le long processus de collaboration

9. Cette vision a surtout été véhiculée par des écrits ultérieurs à 9 Evenings.

10 Pontus Hultén, Frank Königsberg (dir.), 9 Evenings: Theatre and Engineering, New York, Experiments in Art and Technology, The Foundation for Contemporary Performance Arts, 1966.

11. Simone Whitman, «Theater and engineering : an experiment : notes by a participant», in Artforum 5, n 6, fév. 1967, p. 29. 
et d'échange entre artistes et ingénieurs ${ }^{12}$. Preuve de leur importance, les diagrammes sont publiés dans le programme distribué aux spectateurs et deviennent l'affirmation visuelle du propos de ce festival, le symbole de la rencontre entre l'artiste et l'ingénieur.

Les artistes ayant été individuellement impliqués dans la réalisation des diagrammes, il est possible d'y lire en filigrane quelle est leur propre conception de la technologie dans le contexte du spectacle vivant. Pour John Cage et Alex Hay, la technologie permet d'amplifier et de rendre perceptibles des phénomènes habituellement inaudibles. Qu'il s'agisse des sons des ondes cérébrales ou des muscles (Hay) ou de «tous les sons pouvant être captés au moment de la performance ${ }^{13} »$ (Cage), l'enjeu est d'augmenter la perception du spectateur. On retrouve cet aspect dans la performance de Rauschenberg, où l'utilisation de caméras infrarouges rend visible une foule présente sur scène, évoluant dans l'obscurité. Pour Tudor, le dispositif technologique est à la fois un instrument et une partition musicale à interpréter dans le temps de la performance. La transformation d'un média en un autre est une dimension explorée par Robert Rauschenberg (le son des raquettes en commande des lumières), Lucinda Childs (le mouvement en son, le son en image) et David Tudor (le son en variation lumineuse et en image). La commande à distance des différents éléments de la représentation (lumières, son, plateformes), afin de leur conférer un mouvement, caractérise la chorégraphie de Deborah Hay. Lucinda Childs, John Cage et David Tudor s'attachent à la possibilité d'insuffler un comportement aléatoire et imprévisible aux objets. Quant à Yvonne Rainer, c'est la notion de programmation qui apparait dans son diagramme : conçue comme un programme séquentiel mémorisé, la boîte noire permet de déclencher une suite d'actions dans un ordre défini au préalable. La proposition de Paxton, dont le diagramme présente les différents composants de la performance en fonction du parcours du spectateur, est une tentative d'intégration de ce dernier dans le dispositif lui-même : ce n'est que par son déplacement que le spectateur peut expérimenter différentes situations d'immersion (corporelle, visuelle et auditive) et interagir avec le dispositif. Whitman a recours à la technologie pour créer une régie image et son qui lui permet de mixer en direct de multiples sources vidéo et audio. Enfin, la vision de Fahlström est sans aucun doute la plus classique : en régie, à l'abri du

12. Pour un résumé des différentes étapes et les réactions des uns et des autres, cf. Simone Whitman, ibidem, pp. 26-30. Billy Klüver a également écrit plusieurs textes sur ce sujet, notamment : «Theater and Engineering : An Experiment 2. Notes by an Engineer». Artforum 5, n 6, fév. 1967, pp. 31-33 ; «The Great Northeastern Power Failure». College Art Association, Annual Meeting, New York, 1966. Re-publié in Randall Packer, Ken Jordan (dir.), Multimedia : From Wagner to Virtual Reality, New York, W. W. Norton, Washington, Zakros InterArts, 2001, pp. 33-38. De nombreux témoignages sont également réunis dans les archives conservées in Experiments in Art and Technology. Records, 1966-1993, Getty Research Institute, Research Library, Accession no. 940003 ; fonds 9 Evenings: Theatre and Engineering, Fondation Daniel Langlois pour l'art, la science et la technologie.

13. Cit. in Pontus Hultén, Frank Königsberg (dir.), 9 Evenings : Theatre and Engineering, op. cit. 
regard du public, tous les éléments convergent vers un tableau de commande qui centralise toutes les fonctions.

Un constat s'impose : un même système n'implique pas un même usage, ni une même conception des technologies et encore moins une esthétique. C'est sans doute là le tour de force de 9 Evenings et son message le plus important pour les expérimentations artistiques qui vont suivre. Ceci est dû à la conception d'un système qui ne se veut pas figé, mais assemblage d'éléments en fonction du besoin de chaque artiste : décodeurs, relais, amplificateurs, etc. sont combinés de manière différente, ce qui apparaît très clairement dans les diagrammes. Car ce qui est ici proposé aux artistes, c'est bien une combinatoire d'éléments de base, auxquels ils peuvent ajouter des éléments spécifiques. Bien souvent, ces éléments spécifiques sont les plus visibles et apparaissent comme étant les plus significatifs de la performance. On associe ainsi les raquettes à la performance de Rauschenberg, les plateformes mobiles à celle de Deborah Hay, le Ground Effect Machine et le Sonar à celle de Lucinda Childs, etc. Certes, il a fallu relever de nombreux défis pour mettre au point ces machines, et elles sont souvent remarquables, mais elles demeurent les accessoires (au sens théâtral) d'un système qui soustend toutes les performances : le TEEM (Theater Electronic Environmental Module), ou encore THEME (Theater Environmental Modular Electronic), dont Billy Klüver ne cesse de vanter à juste titre le caractère novateur.

Le TEEM est composé de 250 à 300 pièces, selon les descriptions qui en sont faites : décodeurs, encodeurs, amplificateurs, commandes de tonalité, relais électriques, console son, «Proportional Control System », récepteurs et émetteurs FM, cellules photo-électriques, hautparleurs, commutateurs à tambour, préamplificateurs. La plupart de ces unités électroniques sont placées dans la régie, considérée comme une «boîte noire » par les ingénieurs. Elles permettent de contrôler à distance les éléments placés sur scène (lumières, haut-parleurs, caméras, microphones, projecteurs d'image, moteurs, etc) et sont reliées à ces derniers soit par des câbles, soit par un système sans fil. Il ne s'agit pas tant d'élaborer une scénographie, que de mettre en réseau les différents éléments du spectacle, de créer un "environnement» électronique, et d'élaborer des interfaces entre le système et les performeurs et/ou les techniciens.

En permettant de visualiser la combinatoire des éléments du TEEM propre à chaque performance, la réalisation des diagrammes fait surgir un autre problème : «Un seul coup d'œil aux dix diagrammes suffisait pour comprendre que les changements entre les artistes 
une fois par soir pourraient prendre des heures ${ }^{14}$. » Les diagrammes entraînent la réorganisation du matériel. Afin de simplifier et de systématiser l'approche, Herb Schneider propose d'utiliser un tableau de commande qui permet de connecter tous les éléments les uns aux autres : le AMP Equipment, prêté par Amp. Inc. of Harrisburg, Pa. Cet « équipement» permet de programmer et de mémoriser les commandes pour chacune des performances : un panneau de raccordement sur lequel sont établies toutes les connexions est inséré dans un lecteur connecté à chacun des éléments du TEEM. Ce choix a par la suite été très critiqué, car des erreurs de câblage ont fortement perturbé les performances, surtout les premiers jours de la manifestation. Ces erreurs sont essentiellement le fait d'un manque de temps suffisant pour vérifier et tester tout le matériel.

Si l'on ne peut à proprement parler d'informatique, les diagrammes, et le recours au AMP Equipment qui en découle, mettent en évidence l'utilisation de principes et de logiques informatiques dans un contexte de spectacle vivant et de technologies analogiques: programmation, mémorisation, transformation d'un média en un autre, logique aléatoire, combinatoire, etc. Les ingénieurs et les artistes impliqués dans 9 Evenings ont pressenti l'impact de l'informatique sur le spectacle vivant, bien au-delà de l'équipement technique des régies ${ }^{15}$. La collaboration artiste-ingénieur et les équipes multidisciplinaires, la création d'un environnement scénique interactif, la recherche d'une perception augmentée, la commande à distance et sans fil, ou encore la génération du son par le mouvement dansé sont autant d'axes développés aujourd'hui dans le spectacle vivant qui se confronte aux technologies numériques.

Aujourd'hui, 9 Evenings constitue une référence incontournable des relations entre artistes et ingénieurs, en particulier d'un point de vue méthodologique pour les processus de création. Pour ne donner qu'un seul exemple récent, la programmation 2012 de la biennale ZERO1 à San José s'inscrit explicitement dans la lignée de 9 Evenings : «Le programme 2012 des spectacles de la Biennale ZERO1 considère 9 Evenings : Theatre and Engineering comme un point de repère pour découvrir des exemples contemporains d'artistes qui explorent les nouvelles technologies dans les arts de la scène ${ }^{16}$. 19 Evenings a indéniablement influencé la création de certains structures culturelles dédiées aux relations entre art et technologies. Il existe notamment un lien direct entre 9 Evenings et la naissance de l'IRCAM : en 1974, Pierre

\footnotetext{
14. Herb Schneider, "A Glimpse or More at Some Technical Aspects Not Seen by the Third Partner of Nine Evenings - The public », Experiments in Art and Technology. Records, 1966-1993, Getty Research Institute, Research Library, Accession no. 940003

15. Le premier jeu d'orgue informatisé a été installé au Vivian Beaumont Theater du Lincoln Center, en 1965.

16. http://2012.zerolbiennial.org/Performances (consulté le 8 septembre 2014).
} 
Boulez engage comme premier conseiller scientifique Max Mathews, qui avait fait partie des ingénieurs mis à disposition par Bell labs en 1966.

Certaines tentatives de reconstitutions, ou plutôt de réinterprétation de certaines œuvres avec des technologies contemporaines ont également eu lieu. La performance de John Cage, Variations VII, a connu au moins trois versions récentes : en 2007, à l'occasion de la présentation de l'exposition 9 evenings reconsidered et du colloque international re:place (Histories of Media, Art, Science and Technology), par Hans W. Koch et Volker Straebel ; en 2008, lors de l'AV festival à Newcastle, par Atau Tanaka en collaboration avec Matt Wand et les membres du collectif Zoviet France ; en 2007 et en 2008 par le Mobius Artists Group à Boston $^{17}$. Les téléphones, les radios, les multiples éléments du dispositif qui permettaient dans la version originale de jouer avec «tous les sons pouvant être captés au moment de la performance ${ }^{18} \gg$ sont remplacés par des téléphones cellulaires, des connexions Internet ou encore des flux audio transitant par des satellites.

Finalement, c'est davantage le modèle de l'artiste en résidence dans une entreprise qui s'est développé, tel qu'à pu le penser le programme Art \& Technology (A\&T) mis en place à partir de 1967 par Maurice Tuchman au Los Angeles County Museum of Art (LACMA). Le programme Collide du CERN, ou encore le laboratoire arts-sciences du CNES, lequel permet aux artistes d'effectuer des expériences dans des vols paraboliques ${ }^{19}$, sont dans cette lignée. L'une des originalités de 9 Evenings est d'avoir proposé à un groupe d'artistes de collaborer sur le moyen terme avec les ingénieurs de l'une des entreprises les plus innovantes au monde. Ce type de collaboration, et encore plus à cette échelle, demeure une exception, qui plus est dans le champ du spectacle vivant.

\footnotetext{
17. Sur l'interprétation de Variations VII par le Mobius Artists Group, cf. David P. Miller, « Indeterminacy and performance practice in Cage's variations "), in American Music 27, n 1, 2009, pp. 60-86.

18. Cit. in Pontus Hultén, Frank Königsberg (dir.), 9 Evenings : Theatre and Engineering, op. cit.

19. Le chorégraphe Gilles Jobin a été le premier Lauréat du programme Collide en 2011. La chorégraphe Kitsou Dubois et le performeur Marcel.li Antúñez ont bénéficié du programme du CNES.
} 


\section{Illustrations}

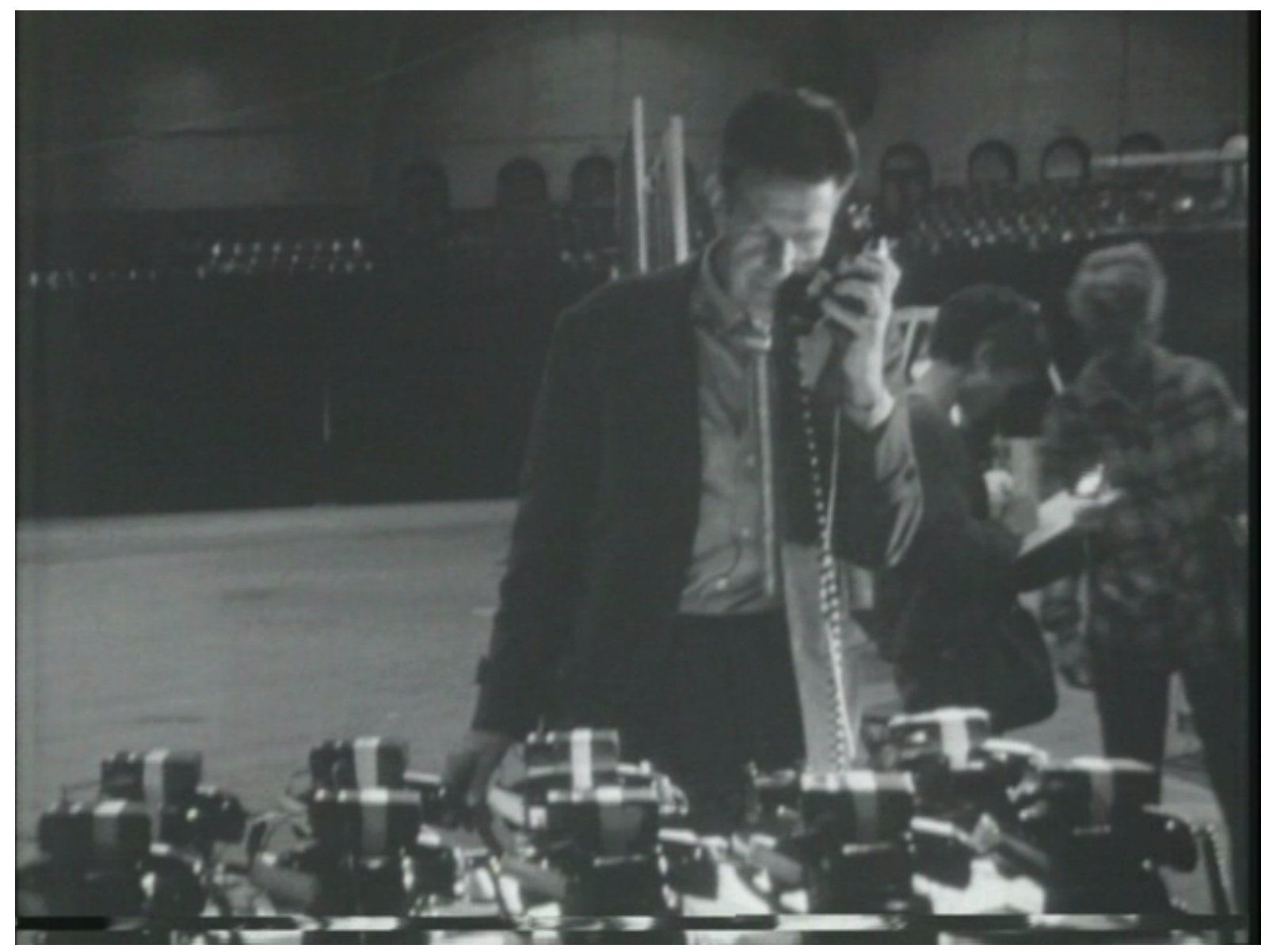

John Cage testant les lignes téléphoniques permettant de retransmettre en direct des sons captés à l'extérieur de la salle pour Variations VII. Photogramme issu des captations tournées par Alfons Schilling dans le cadre de 9 Evenings: Theatre \& Engineering, The 69th Regiment Armory (New York, N.Y., États-Unis), octobre 1966. (C) Alfons Schilling. Gracieuseté de Julie Martin (Experiments in Art and Technology), de la succession Alfons Schilling et de la Fondation Daniel Langlois. 


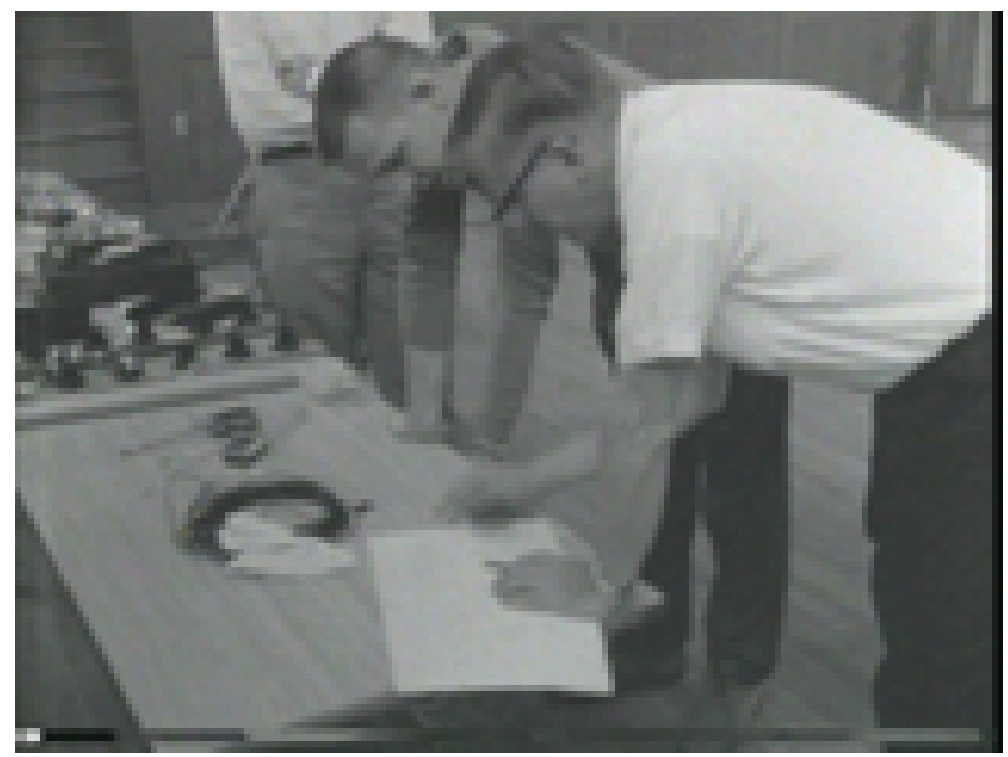

Ingénieurs au travail pendant les séances de préparation au gymnase de Berkeley en septembre 1966. Photogramme issu des captations tournées par Alfons Schilling dans le cadre de 9 Evenings: Theatre \& Engineering, The 69th Regiment Armory (New York, N.Y., ÉtatsUnis), octobre 1966. (C) Alfons Schilling. Gracieuseté de Julie Martin (Experiments in Art and Technology), de la succession Alfons Schilling et de la Fondation Daniel Langlois. 


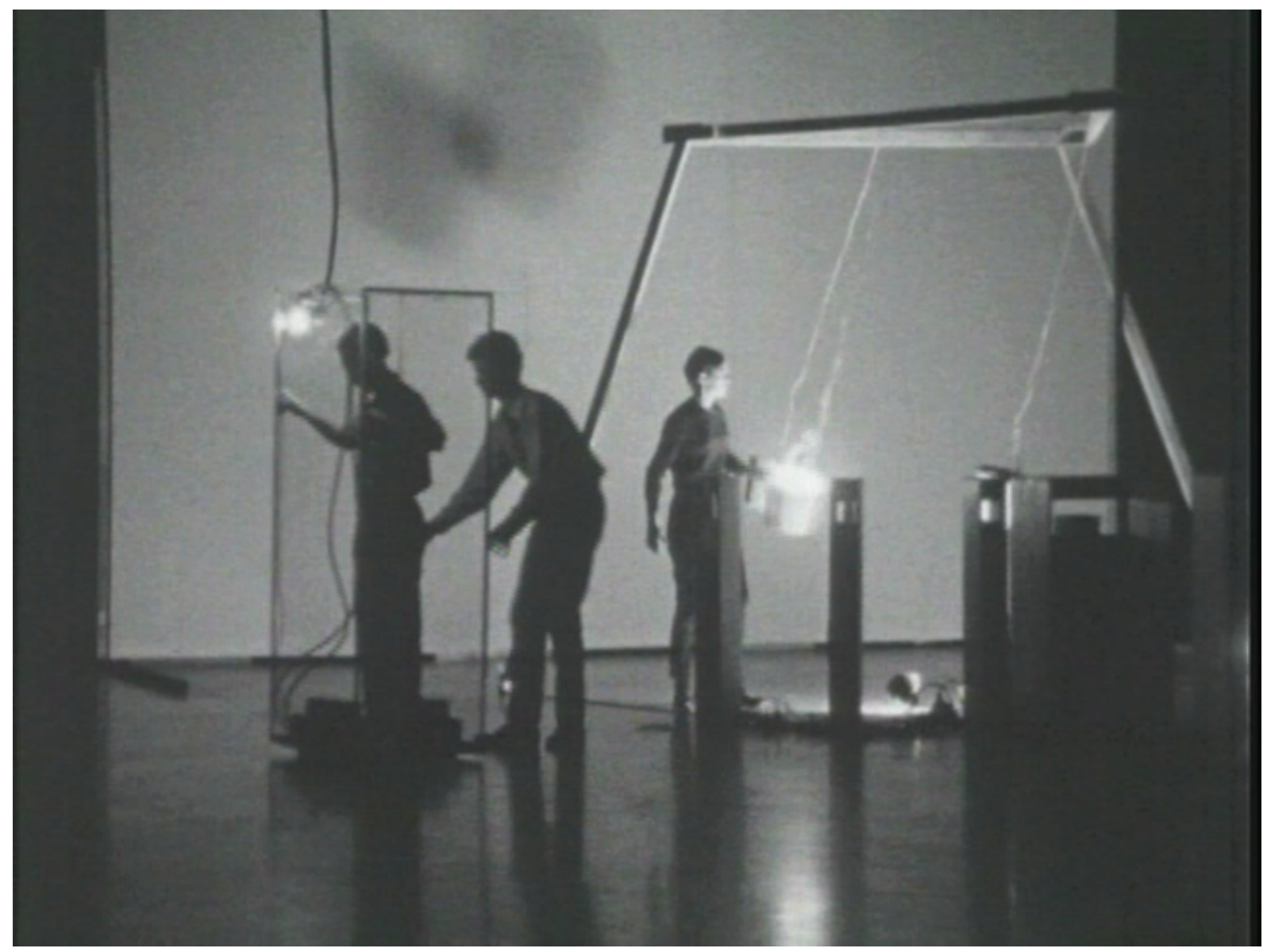

Lucinda Childs, Vehicle. Alex Hay, William Davis et Lucinda Childs. A gauche, le Ground Effect Machine ; à droite, le sonar et la structure qui supporte les seaux suspendus. En arrière plan, un écran blanc qui reçoit l'ombre projetée de la scène. Photogramme issu des captations tournées par Alfons Schilling dans le cadre de 9 Evenings: Theatre \& Engineering, The 69th Regiment Armory (New York, N.Y., États-Unis), octobre 1966. (C) Alfons Schilling. Gracieuseté de Julie Martin (Experiments in Art and Technology), de la succession Alfons Schilling et de la Fondation Daniel Langlois. 